\title{
Time variations of water masers
}

\author{
Tarja Liljeström \\ Helsinki University of Technology, Metsähovi Radio Observatory, \\ Otakaari 5.A, FIN-02150 Espoo, Finland
}

\author{
Cari R. Gwinn \\ University of California, Physics Department, Broida Hall \\ Santa Barbara, CA 93106, USA
}

\begin{abstract}
The strong water maser line at $22 \mathrm{GHz}$ is an excellent tool for studying shocked and turbulent interstellar regions, especially, if simultaneous single-dish and VLBI data are available. After a brief review of $22 \mathrm{GHz}$ time variation studies, we focus on effects caused by magnetic field pressure on observed properties of water masers. We use the powerful and rich maser cluster W49N as an example. Furthermore, we point out the connection between postshock wave damping and observed excess in single-dish flux density and line width.

Keywords: - ISM: individual (W49N) - ISM: magnetic fields - masers shock waves - turbulence - waves.
\end{abstract}

\section{Introduction}

Early water maser observations (e.g., Knowles et al. 1969; Meeks et al. 1969) indicated that $22 \mathrm{GHz}$ water masers are strongly variable. Usually, the flux density of a maser feature remains constant for long periods, then increases rapidly, remains at high level for a time, and then returns to about the original level. We term such short-lived, dramatic increases in flux density "outbursts". Observations during the past 30 years have shown that the time scales of the flux density variations range from about one week to years. Most variability studies have concentrated on time variations of the flux density and explained these as following from changes in pump power, gain, or degree of saturation (e.g., Sullivan 1971, 1973; Genzel \& Downes 1977; Garay, Moran, \& Haschick 1989; Abraham \& Vilas Boas 1994). Correlated variability of $22 \mathrm{GHz}$ masers has been discussed by Gammon (1976), White (1979), and Liljeström (2000), while the long-term variability has been addressed, e.g., by Lekht, Marquez, \& Sorochenko (1995), Lekht, Marquez, \& Mendoza-Torres (1996), and Berulis, Lekht, \& Mendoza-Torres (1996). Variations observed in the line velocity of maser features are generally small (e.g., Sullivan 1971; 1973; Liljeström \& Gwinn 2000) and they have been explained as resulting from hydrodynamic turbulence or clumpiness (e.g., Gwinn 1994a; Lekht, Mendoza-Torres, \& Silantev 1999).

We have recently analysed $22 \mathrm{GHz}$ single-dish data of some 150 maser outbursts (Liljeström, 2000) and simultaneous VLBI observations of W49N (Gwinn, 
Moran, \& Reid 1992; Gwinn 1994a, 1994b) to infer physical conditions in these strongly shocked regions (Liljeström \& Gwinn 2000). We found two groups of masers, which behaved differently in a statistical sense. The intense masers within the Doppler velocity range of the dense ambient medium (as traced by $\left.\mathrm{C}^{34} \mathrm{~S}[5-4]\right),-2<V_{L S R}<18 \mathrm{~km} \mathrm{~s}^{-1}$, were termed "inside masers"; masers outside this velocity range were termed "outside masers". These two maser categories could also be called low-velocity masers and high-velocity masers, respectively. With our databases we were able to fix the free parameters in the shock model of Hollenbach \& McKee (1989) and the maser model of Elitzur, Hollenbach, \& McKee (1989). This enabled a straightforvard determination of some 20 shock and maser parameters in W49N (Liljeström \& Gwinn 2000). This study showed the importance of the magnetic field in masing regions. Therefore, we here focus on dependences of observed flux density, line velocity variations, line width, and space velocity of maser features on different magnetic pressure components.

\section{Dependence of Peak Flux Density on Magnetic Pressure}

Water masers frequently form when supersonic winds from young stellar objects strike dense surrounding material thereby exciting shocks. When shocks weaken they become large-amplitude hydromagnetic waves, which consist of compressive magnetosonic waves and of noncompressive Alfvén waves. When the interstellar medium contains a well-coupled magnetic field, disturbances propagate with the Alfvén velocity, $V_{A}=B /(4 \pi \rho)^{0.5}$, where $B$ is the magnetic field strength and $\rho$ the mass density of the medium. The idea that interstellar magnetic fields are coupled to gas motions was first discussed by Alfvén (1943). Chandrasekhar \& Fermi (1953) first suggested that the pressure associated with cloud magnetic fields provides cloud support against gravity.

The total magnetic pressure of a medium consists of a static field pressure component, $B^{2} / 8 \pi$, and a wave pressure component, $P_{w}=\delta B^{2} / 8 \pi$, where $\delta B$ is the field fluctuation directed perpendicularly to $B$ (e.g., McKee \& Zweibel 1995). Due to equipartition between kinetic energy and potential energy of undamped Alfvén waves, the wave pressure can equally well be expressed as, $P_{w}=(1 / 2) \rho \cdot \delta V_{B}{ }^{2}$, where $\delta V_{B}$ is the velocity fluctuation perpendicular to the field lines. The ratio of wave pressure to static field pressure is thus (McKee \& Zweibel 1995),

$$
(1 / 2) \rho \cdot \delta V_{B}^{2} /\left(B^{2} / 8 \pi\right) \equiv \delta V_{B}^{2} / V_{A}^{2},
$$

where $V_{A}$ is the Alfvén velocity. For wave fluctuations this ratio is expected to be smaller than one, because higher wave amplitudes would create shocks (McKee \& Zweibel 1995). From equation (1) it is clear that the wave pressure is traced by $\delta V_{B}{ }^{2}$ while the static field pressure is traced by $V_{A}{ }^{2}$.

The preshock Alfvén velocity is defined by $V_{A}$ (pre) $=1.84 \mathrm{~km} \mathrm{~s}^{-1} b$ (Hollenbach \& McKee 1989), where $b$ is the magnetic field parameter, $B_{o} / n_{o}{ }^{0.5}$, and $n_{o}$ the preshock density of hydrogen nuclei. Because the maser thickness, preshock density, and space/shock velocity of the maser features were known from observations, we determined $b$ from equation (10) of Liljeström \& Gwinn (2000) (originally from Hollenbach \& McKee 1989).

The postshock Alfvén velocity is defined by $V_{A}$ (post) $=1.84 b\left(n / n_{o}\right)^{0.5}$ (Hollenbach \& McKee 1989), where $n$ is the postshock hydrogen nuclei den- 

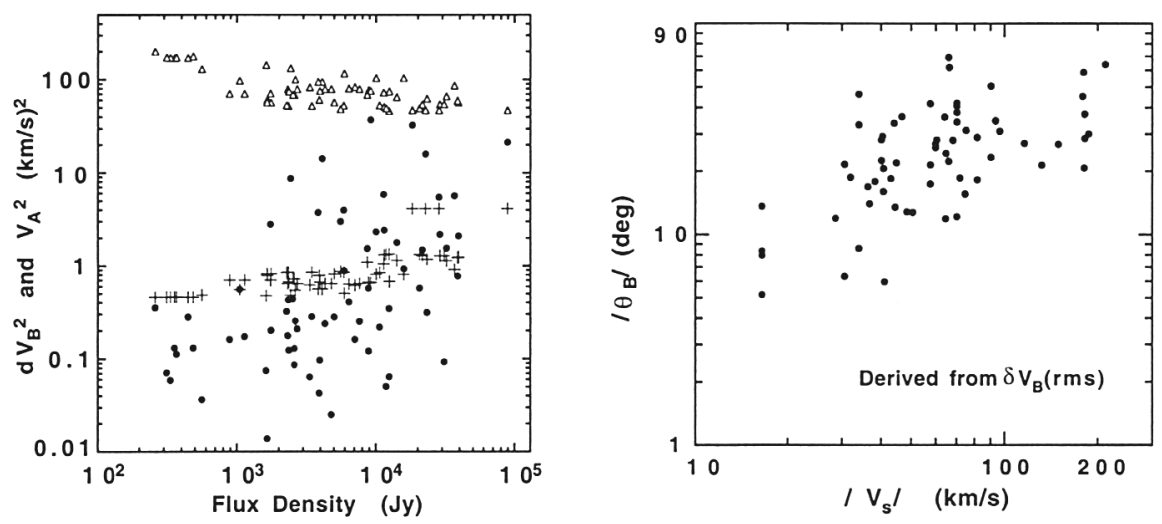

Figure 1. (Left) Single-dish peak flux density of maser outbursts in W49N as a function of preshock Alfvén velocity in quadrature, which traces preshock static magnetic field pressure. (Right) Same as the left panel but as a function of space/shock velocity of masers, which traces the postshock static field pressure.

sity. Using equations (9) and (11) of Liljeström \& Gwinn (2000), the relation, $V_{A}{ }^{2}$ (post) $=1.41 V_{A}$ (pre) $V_{s}$, results, where $V_{s}$ is the space/shock velocity of masers and the specification 'post' ('pre') refers to conditions within (outside) the masing region. Because in the space velocity range $28-212 \mathrm{~km} \mathrm{~s}^{-1}$ of W49N, the change in $V_{A}$ (pre) is only some $0.5 \mathrm{~km} \mathrm{~s}^{-1}$, the postshock static field pressure depends mainly on the space/shock velocity of masers.

Figure 1 shows that the peak flux density of maser outbursts, $F_{\text {max }}$, increases with preshock static field pressure, but decreases with space velocity, i.e., with postshock static field pressure within masers. The biggest maser flares require thus a large preshock static magnetic field pressure but a small postshock static field pressure (i.e., a small space velocity).

\section{Line Velocity Variations during Maser Outbursts}

Single-dish observations reveal small variations in the Doppler velocity of a maser feature during outbursts (e.g., Liljeström \& Gwinn 2000). Over some period of time, these "turbulent" velocities cause a reorganization of the masing gas and thus a change in the maximum gain along line of sight. As a consequence the Doppler velocity of the maximum intensity changes. In $\mathrm{W} 49 \mathrm{~N}$ this turbulent velocity within a maser feature is much less than the turbulent velocity between maser features on larger scales (Gwinn 1994a).

Figure 2 (left) shows a few examples of line velocity variations of maser outburst features in $\mathrm{W} 49 \mathrm{~N}$ as a function of time. A quantitative measure of the observed line velocity variation, $\delta V_{o b s}$, is the maximum shift in Doppler velocity of a feature during an outburst. Figure 2 (top panel, right) reveals that the line velocity variations are nonthermal. According to the middle panel, the velocity shift of outside masers may increase slightly with decreasing preshock static field pressure. We note, however, that the relative error in $V_{A}^{2}$ (pre) 

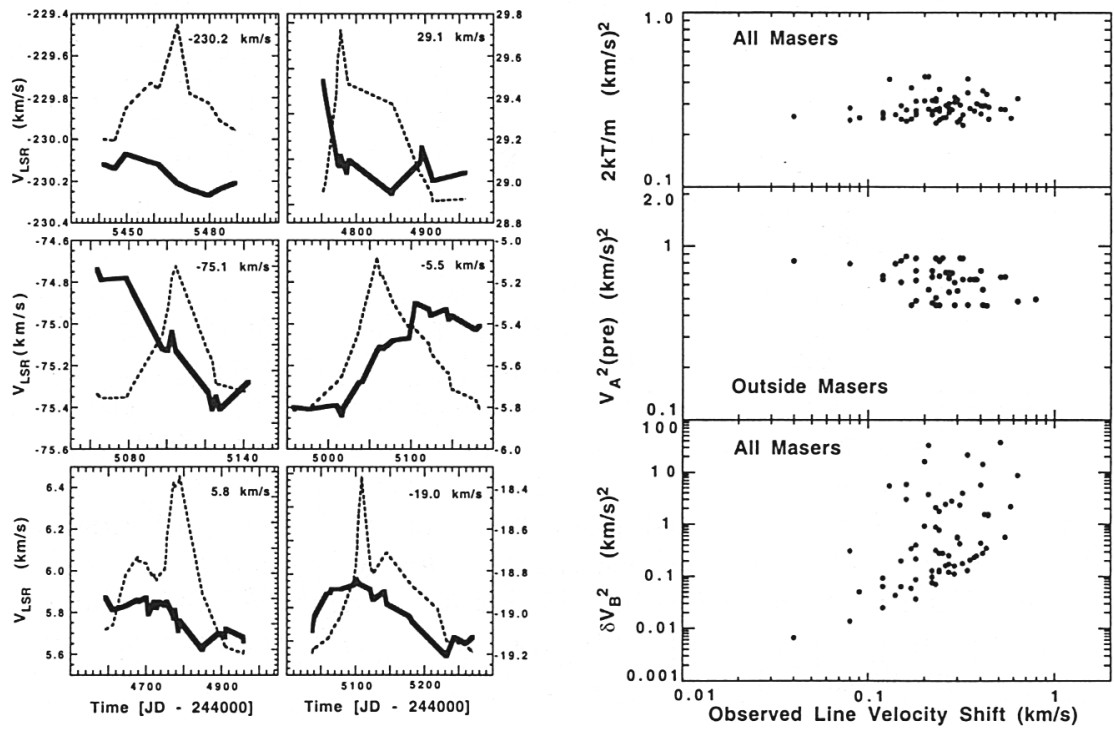

Figure 2. (Left) Examples of line velocity variations as a function of time (thick line) superimposed on the corresponding flux density curve. The error in $V_{L S R}$ is $0.012 \mathrm{~km} \mathrm{~s}^{-1}$. (Right) Relations between observed line velocity shift and thermal velocity dispersion in quadrature (relative error 26\%), preshock static field pressure as traced by $V_{A}{ }^{2}$ [pre] (relative error 50\%), and Alfvén wave pressure as traced by $\delta V_{B}^{2}$ (relative error $46 \%$ ).

is $50 \%$. The bottom panel (Fig. 2, right) shows a strong dependence of the observed line velocity shift on the wave pressure, as expected from the following argumentation.

Because the wave fluctuation, $\delta V_{B}$, of an Alfvén wave is oriented perpendicular to magnetic field lines, the relation between $\delta V_{B}$ and observed line velocity shift, $\delta V_{o b s}$, is obtained from,

$$
\delta V_{B}=\delta V_{o b s} / \sin \theta_{B}
$$

where $\theta_{B}$ is the inclination of the magnetic field to the line of sight. Because strong shocks tend to be perpendicular shocks (i.e., the shock/space velocity is perpendicular to field lines; see Hollenbach \& McKee 1979), the field inclination, $\theta_{B}$, after strong shocks can observationally be determined from the inclination of the space velocity vector of masers to the line of sight, $\theta_{V}$, obtained from VLBI proper motion measurements of maser features:

$$
\theta_{B}=90^{\circ}-\theta_{V} .
$$

Combining equations (2) and (3) gives the wave fluctuation, $\delta V_{B}$, and thus the Alfvén wave pressure, $\rho \cdot \delta V_{B}{ }^{2} / 2$, after strong shocks. As expected from equation (2), the observed line velocity shift correlates with wave pressure, however, 

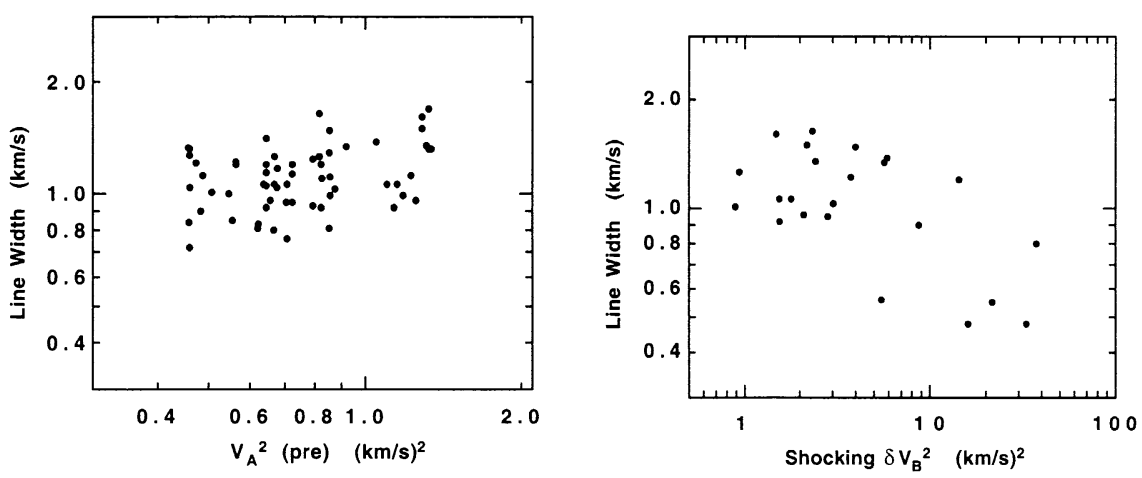

Figure 3. (Left) Observed single-dish maser line width at outburst maxima as a function of postshock thermal pressure as traced by the thermal velocity dispersion in quadrature. (Right) Same as left panel but as a function of shocking wave pressure as traced by $\delta V_{B}{ }^{2}$.

only as long as the wave fluctuation is nonshocking. For shocking wave pressures $\left(\delta V_{B}^{2}>V_{A}^{2}\right.$ [pre]), no correlation between observed line velocity shift and Alfvén wave pressure exists (see upper part of the bottom right panel of Fig. 2).

The observed Doppler velocity shift of maser outburst features can be used to determine the inclination of the preshock rms field to the line of sight. Substituting $\delta V_{B}(\mathrm{rms})=(1 / 2)^{0.5} V_{A}$ (pre) into equation (2), the complete single-dish sample of outside masers (125 outbursts), gives a median of $\theta_{r m s}=-32^{\circ}\left( \pm 2.3^{\circ}\right)$. In our data set the median rms field inclination of outside masers, $-32^{\circ}$, is roughly perpendicular to the median inclination of the space vectors of outside masers to the line of sight, $+49.7^{\circ}$, as expected for strong shocks.

For inside masers the median preshock rms field inclination to the line of sight is $\theta_{r m s}=-18^{\circ}\left( \pm 2.6^{\circ}\right)$. The smaller rms field inclination of inside masers follows from the typically 2 - 4 times larger preshock densities of inside masers when compared to outside masers (Liljeström \& Gwinn 2000). As a consequence, inside masers have typically 2 - 4 times higher preshock magnetic field strengths and thus 1.5 - 2.8 times larger preshock Alfvén velocities than outside masers. These larger Alfvén velocities (and thus larger $\delta V_{B}[\mathrm{rms}]$ ) account for the smaller rms field inclinations obtained for inside masers (eq. [2]).

\section{Maser Line Width at Outburst Maxima}

A number of authors have considered factors that contribute to line widths of masers. Line width narrowing of unsaturated masers and rebroadening back to Doppler widths due to radiative transfer and saturation effects have been discussed in classic papers of maser theory (e.g., Litvak 1970; Goldreich \& Kwan 1974). However, besides radiative transfer effects, also pressure effects may contribute to maser line widths as pointed out in the following.

Figure 3 (left) displays the observed single-dish line width at outburst maxima as a function of the thermal velocity dispersion (in quadrature) of water, $2 k T / m$, which traces the thermal pressure, $(1 / 2) \rho(2 k T / \mu)$, where $\mu=2.33$ amu 
is the mass of the mean mass particle. The postshock kinetic temperature has been determined from equation (13) of Liljeström \& Gwinn (2000), which is observationally constrained through the measured space velocity of maser features, the single-dish line width at outburst maxima, and preshock density (Serabyn, Güsten, \& Schulz 1993). Interestingly, in contrast to thermal lines, maser line widths tend to decrease slightly with increasing thermal pressure. Probably this is a consequence of the much stronger dependence of maser line width on shocking wave pressure, $\left(\delta V_{B}{ }^{2}>V_{A}{ }^{2}\right.$ [pre]) as shown in Figure 3 (right).

This surprising line width behavior can be explained with the argumentation of McKee \& Zweibel (1995): Behind a shock, the kinetic contribution to the wave energy is smaller than the magnetic contribution by the compression factor of the shock. Therefore, the kinetic energy becomes negligible just behind a strong shock and a finite time is needed to establish equipartition between kinetic and magnetic energy. This is seen in the pressure components obtained for the different maser categories of W49N. For outside masers (typical compression factor 200) the wave pressure is over two orders of magnitude smaller than the static field pressure and one order of magnitude less than the postshock (onedimensional) thermal pressure (which is of the order of $10^{-6} \mathrm{~N} \mathrm{~m}^{-2}$ for all maser categories). For inside masers (excluding the big flare feature reported by Liljeström \& Gwinn 2000) the static field pressure is still some 37 times larger than the wave pressure, which is of the order of the postshock thermal pressure. However, for the big flare feature (with a compression factor of only some 15) the (shocking) wave pressure is one third of the static field pressure and, most noteworthy, about six times larger than the postshock thermal pressure (Liljeström \& Gwinn 2001). Thus, for big maser flares the postshock thermal pressure is irrelevant as compared to the wave pressure.

\section{Flux Density Excess and Line Width Excess}

At outburst maxima the water masers in $\mathrm{W} 49 \mathrm{~N}$ show a single-dish peak flux density, $F_{\text {max }}$, typically about twice as large as the actual increase in flux density during an outburst, $\Delta F_{\max }$. The reason for these two different values is that the flux density level at start of an outburst often deviates from the level at the end of an outburst (for a schematic illustration of a typical maser outburst see Fig. 4 of Liljeström \& Gwinn 2000). This flux density excess, $F_{\max }-\Delta F_{\max }$, is strongest for inside masers (Fig. 4, left), which also show the largest wave pressures. Besides the flux density excess present in the single-dish data, a flux density excess exists also between single-dish and VLBI flux densities of W49N (Walker, Matsakis, \& Garcia-Barreto 1982; Gwinn 1994c). Gwinn (1994c) explained this flux density excess as resulting from coherent scattering of maser radiation in ambient dense plasma of the host HII region. He termed these scattering regions maser halos, which he estimated to have typical sizes of about $30 \mathrm{AU}$. Because the halo had about the same flux density as the corresponding compact maser feature, he concluded that the scattering material must have an optical depth about 1 , which is best matched for coherent scattering by ion sound waves in a single shock.

The single-dish flux density excess (if attributed to damping waves) agrees with predictions (McKee \& Zweibel 1995) according to which the energy density of a wave is substantially increased upon crossing a radiative shock, with up 

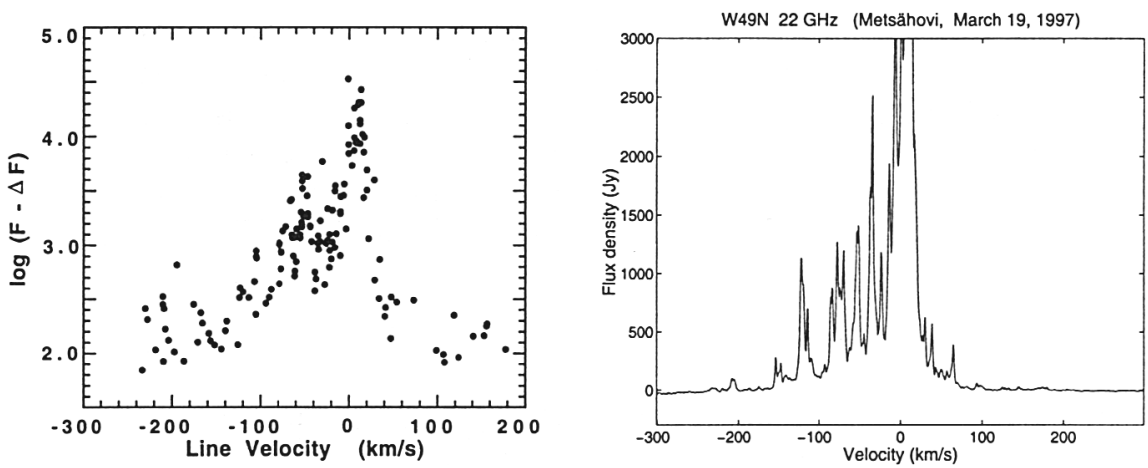

Figure 4. (Left) Observed flux density excess as a function of Doppler velocity of maser features in W49N. (Right) Broad-band spectrum of W49N.

to half of it going into magnetosonic waves. These slow magnetosonic waves propagate perpendicularly to the field lines and contribute to the anisotropy of the wave pressure (Sridhar \& Goldreich 1994; Goldreich \& Sridhar 1995, 1997).

The observed line width excess can be used to evaluate the nature of the wave damping. For a linear damping process like scattering, the scatterers should have about the same velocity dispersion as the compact maser feature. Therefore, the single-dish line width (in quadrature) should be some two times the line width of the compact maser feature (in quadrature). The median VLBI line width of inside masers and outside masers is 0.52 and $0.80 \mathrm{~km} \mathrm{~s}^{-1}$, respectively (Gwinn 1999). Thus, in presence of a linear wave damping, the corresponding single-dish line width should be 1.13 (outside masers) and $0.74 \mathrm{~km} \mathrm{~s}^{-1}$ (inside masers). Compared to the actually observed median single-dish line width of outside masers $\left(1.10 \pm 0.02 \mathrm{~km} \mathrm{~s}^{-1}\right)$ and inside masers $\left(1.26 \pm 0.02 \mathrm{~km} \mathrm{~s}^{-1}\right)$, the agreement for outside masers is excellent. However, the line width excess of inside masers, together with the strong dependence of the flux density excess on postshock magnetic field inclination (Liljeström \& Gwinn 2001), suggest that nonlinear damping (responsible for the anisotropic wave pressure) is important for inside masers. Nonlinear wave-wave interactions among shear Alfvén waves are frequently produced in turbulent cascades of wave energy from one scale to another (Sridhar \& Goldreich 1994; Goldreich \& Sridhar 1995, 1997). An important role in the energy dissipation of this Alfvénic turbulence play short-lived Alfvén waves which couple to slow sonic waves.

Experiments and simulations of turbulent flows show that vorticity and high energy dissipation are generally concentrated in coherent, "thin-tube" like structures, a property called internal intermittency (e.g., Münch 1999). Figure 4 (right) shows that the central Doppler velocity range of $\mathrm{W} 49 \mathrm{~N}$ is characterized by a broad emission component. The existence of such a broad flux density component is generally termed integral kurtosis of turbulent flows. Together with the observed excesses in flux density and line width, these characteristics support the view that the turbulence in $\mathrm{W} 49 \mathrm{~N}$ is intermittent. Because the 
energy dissipation of intermittent turbulence occurs in burst-like phenomena, it contributes to the complexity of the observed time variations of water masers.

Acknowledgements. T. L. thanks the Institute for Theoretical Physics (ITP) at University of California Santa Barbara for hospitality during the ITP program on Astrophysical Turbulence and acknowledges partial support from the National Science Foundation (USA) under Grant No. PHY94-07194.

\section{References}

Abraham, Z., \& Vilas Boas, J. W. S. 1994, A\&A, 220, 956

Alfven, H. 1943, Ark. Mat. Astron. Fys., 29B, 2

Berulis, I., Lekht, E., Mendoza-Torres, E. 1996, Aston. Reports, 40, 329

Chandrasekhar, S., \& Fermi, E. 1953, ApJ, 118, 113

Elitzur, M., Hollenbach, D. J., \& McKee, C. F. 1989, ApJ, 346, 983

Gammon, R. H. 1976, A\&A 50, 71

Garay, G., Moran, J. M., \& Haschick, A. D. 1989, ApJ, 338, 244

Genzel, R., \& Downes, D. 1977, A\&AS, 30, 145

Goldreich, P., \& Kwan, J, Y. 1974, ApJ, 190, 27

Goldreich, P., \& Sridhar, S. 1995, ApJ, 438, 763

Goldreich, P., \& Sridhar, S. 1997, ApJ, 485, 680

Gwinn, C. R. 1994a, ApJ, 429, 241

Gwinn, C. R. 1994b, ApJ, 429, 253

Gwinn, C. R. 1994c, ApJ, 431, L123

Gwinn, C. R. 1999, in Interstellar Turbulence, ed. J. Franco \& A. Carraminana

(New York: Cambridge Univ. Press), 159

Gwinn, C. R., Moran, J. M., \& Reid, M. J. 1992, ApJ, 393, 149

Hollenbach, D. J., \& McKee, C. F. 1979, ApJS, 41, 555

Hollenbach, D. J., \& McKee, C. F. 1989, ApJ, 342, 306

Knowles, S. H., et al. 1969, Science, 163, 1055

Lekht, E., Marquez, A., \& Mendoza-Torres, E. 1996, A\&AS, 120, 415

Lekht, E., Marquez, A., \& Sorochenko, R. 1995, ApJ, 443, 222

Lekht, E., Mendoza-Torres, E., \& Silantev, N. 1999, Astron. Reports 43, 209

Liljeström, T. 2000, J. Astron. Data, 6, 2

Liljeström, T., \& Gwinn, C. R. 2000, ApJ, 534, 781

Liljeström, T., \& Gwinn, C. R. 2001, ApJ, submitted

Litvak, M. M. 1970, Phys. Rev. A, 2, 2107

McKee, C. F., \& Zweibel, E. G. 1995, ApJ, 440, 686

Meeks, M. L., et al. 1969, Science 165, 180

Münch, G. 1999, in Interstellar Turbulence, ed. J. Franco \& A. Carraminana

(New York: Cambridge Univ. Press), 1

Serabyn, E., Güsten, R., \& Schulz, A. 1993, ApJ, 413, 571

Sridhar, S. \& Goldreich, P. 1994, ApJ, 432, 612

Sullivan, W. T. 1971, ApJ, 166, 321

Sullivan, W. T., 1973, ApJS, 25, 393

Walker, R. C., Matsakis, D. N., \& Garcia-Barreto, J. A. 1982, ApJ, 255, 128

White, G. J. 1979, MNRAS, 186, 377 